\title{
Contribuições da psicometria para a avaliação de respostas psicossociais na enfermagem*
}

\author{
PSYCHOMETRIC CONTRIBUTIONS TO THE ASSESSMENT OF PSYCHOSOCIAL \\ RESPONSES IN NURSING
}

\section{CONTRIBUCIONES DE LA PSICOMETRÍA PARA LA EVALUACIÓN DE RESPUESTAS PSICOSOCIALES EN LA ENFERMERÍA}

\section{Cristiane Giffoni Braga1, Diná de Almeida Lopes Monteiro da Cruz²}

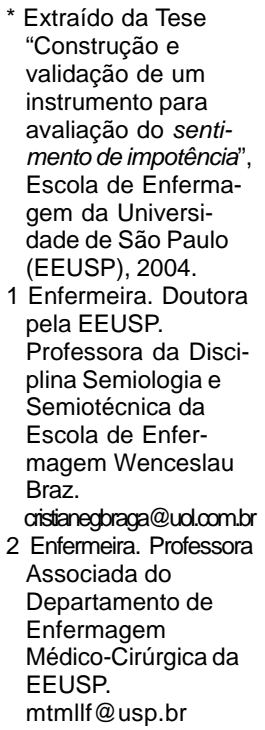

\begin{abstract}
RESUMO
Este artigo apresenta os fundamentos metodológicos que norteiam a construção de instrumentos para avaliação de variáveis psicossociais. Descreve os pólos teórico, empírico ou experimental e analítico e exemplifica o pólo teórico com a experiência do desenvolvimento de um instrumento para avaliar o sentimento de impotência, como um diagnóstico de enfermagem. Os procedimentos mais árduos na construção de medidas são os do pólo teórico. Exigem desenvolver a sensibilidade teórica, exercitandoa num constante ir e vir entre o mais concreto e o mais abstrato; exige desenvolver a tolerância à ambigüidade, sem abandonar o exame das evidências.
\end{abstract}

\section{DESCRITORES}

Diagnóstico de enfermagem. Psicometria.

Metodologia.

\section{ABSTRACT}

This article presents the methodological bases that guide the development of instruments to measure psychosocial variables. It describes the theoretical, empirical or experimental and analytical and exemplifies the theoretical steps through the experience of developing an instrument to measure powerlessness as a nursing diagnosis. The most difficult procedures for developing instruments to are the theoretical steps. They require the development of theoretical sensitivity as a continuous going forward and backward between concrete and abstract issues; they also require the development of tolerance towards ambiguity, but without abandoning the examination of evidences.

\section{KEY WORDS}

Nursing diagnosis.

Psychometrics.

Methodology.

\section{RESUMEN}

Este artículo presenta los fundamentos metodológicos que orientan la construcción de instrumentos para la evaluación de variables psicosociales. Describe los polos teórico, empírico o experimental y analítico y ejemplifica el polo teórico con la experiencia del desarrollo de un instrumento para evaluar el sentimiento de impotencia, como un diagnóstico de enfermería. Los procedimientos más arduos en la construcción de medidas son los del polo teórico. Exigen desarrollar la sensibilidad teórica, ejercitándola en un constante ir y venir entre lo más concreto y lo más abstracto; exige desarrollar la tolerancia a la ambigüedad, sin abandonar el examen de las evidencias.

\section{DESCRIPTORES}

Diagnóstico de enfermería.

Psicometría.

Metodología. 


\section{INTRODUÇÃO}

Respostas psicossociais aos problemas de saúde e processos de vida são freqüentes na clínica de enfermagem. A nominação dessas respostas, processo inerente ao desenvolvimento de sistemas padronizados de linguagem, traz o desafio de tratá-las como construtos. Cada diagnóstico de enfermagem deve ser tratado como um construto para que possa ser compreendido, refinado e útil na prática clínica. Os recursos metodológicos da psicometria são fundamentais para o desenvolvimento dos diagnósticos de enfermagem porque podem auxiliar no refinamento dos construtos que eles representam.

Construtos são abstrações, construções teóricas que objetivam organizar e atribuir significados ao nosso ambiente $^{(1)}$. São exemplos de construtos: ansiedade, dor, sentimento de impotência, desesperança, pesar, medo. Os construtos não podem ser diretamente observados, mas podem ser mensurados pelos atributos ou indicadores derivados da clarificação e definição dos construtos.

A psicometria é, por vezes, aplicada de forma inadequada para o desenvolvimento de instrumentos, sem o cuidadoso escrutínio teórico do construto que o instrumento pretende medir, sem o planejamento e análise cuidadosos dos testes a que o instrumento é submetido. Há proliferação de instrumentos que não são válidos e confiáveis no cenário da saúde ${ }^{(2)}$ e que, por isso, não contribuem para o refinamento do conhecimento. No entanto, com preparação e testes cuidadosos, é possível produzir, na maioria das circunstâncias, instrumentos de medidas de construtos que sejam válidas e confiáveis ${ }^{(3}$.

Este artigo apresenta os fundamentos teórico-metodológicos que norteiam a construção de instrumentos para medida de variáveis psicossociais. Descreve os pólos teórico, empírico ou experimental, e analítico ${ }^{(4)}$, e exemplifica o pólo teórico com a experiência do desenvolvimento de um instrumento para avaliar o sentimento de impotência, como um diagnóstico de enfermagem.

\section{PSICOMETRIA}

A psicometria é um ramo da estatística que estuda fenômenos psicológicos. Foi desenvolvida por estatísticos de formação e, por isso, ainda é definida como um ramo da estatística. Para os psicólogos, ela deve ser concebida como um ramo da psicologia que faz interface com a estatística ${ }^{(5)}$. A psicometria não trata apenas de métodos; ela se insere na teoria da medida que trata da utilização de números no estudo dos fenômenos naturais ${ }^{(5)}$. Medir significa atribuir valores a características ou atributos de um objeto, segundo regras que assegurem a validade e a confiabilidade dos resultados da medida.
Há diversas formas de atribuir valores ou números às características dos objetos. A psicometria fundamenta-se na medida por teoria e para melhor compreendê-la é necessário uma explanação sobre os outros tipos de medida: fundamental e derivada.

Na medida fundamental, o atributo de um objeto pode ser medido diretamente por um outro objeto (instrumento) que tem o atributo que se quer medir. Tome-se como exemplo a medida do comprimento de uma folha de papel utilizando-se uma régua. A unidade de medida da régua é seu atributo de comprimento que pode, por exemplo, ser o centímetro. Esse atributo é o mesmo atributo que se quer medir na folha de papel. Amedida do comprimento do papel será dada pela coincidência de pontos entre a unidade de comprimento marcada no instrumento ${ }^{(5)}$ (o centímetro) e o objeto que se quer medir (o papel). Diz-se, nesse caso, que a régua é uma representação extensiva do atributo comprimento do papel. Amedida fundamental também pode ser chamada de medida direta.

A medida derivada é empregada quando não há um instrumento que possua o mesmo atributo que se quer medir no objeto. Nesses casos, aplicamse as medidas derivadas. Medida derivada é a medida indireta por meio do estabelecimento de uma relação com medidas fundamentais. A medida de densidade é um exemplo de medida derivada. Sabe-se que a massa varia em função de volume e da densidade: massa $=$ volume $\mathrm{x}$ densidade. Como a massa permite medida fundamental (peso, expresso em quilos) e o volume também (o cubo do comprimento $=\mathrm{m}^{3}$ ), então a densidade, que não possui medida fundamental, pode ser medida indiretamente, em função de massa e volume (quilograma dividido por metro cúbico $\left.=\mathrm{kg} / \mathrm{m}^{3}\right)^{(5)}$.

$\mathrm{Na}$ medida por teoria, quando não existem leis relacionando atributos, recorre-se a teorias que fazem hipóteses sobre relações entre os atributos de um fenômeno, permitindo, assim, a medida indireta do seu atributo. Não há como estabelecer uma representação extensiva dos atributos do fenômeno (medida fundamental); também é impossível medilo por meio do estabelecimento de uma relação com medidas extensivas (medida derivada). Na medida por teoria, as concepções teóricas, que explanam as relações entre um fenômeno abstrato e os atributos que lhe dizem respeito, produzem hipóteses sobre o que, teoricamente, poderia ser observado quando o fenômeno ocorre. Tome-se como exemplo o sentimento de impotência como fenômeno de interesse, como o "fenômeno" que se quer mensurar. O sentimento de impotência é um fenômeno altamente abstrato, cujos atributos são teoricamente explanados. Das relações, também teoricamente estabelecidas, entre esses atributos e o sentimento de impotência, podem-se estabelecer hipóteses sobre o que é observado quando uma pessoa se sente impotente. Testes empíricos dessas hipóteses devem produzir resultados 
sobre a validade das relações teoricamente estabelecidas, entre o fenômeno - sentimento de impotência - e seus atributos. A teoria utilizada, que fundamenta as relações fenômenoatributos, deve ser continuamente considerada frente aos resultados dos testes empíricos das hipóteses que dela mesmo se originaram. Ao analisar a teoria frente aos resultados dos testes empíricos, podem surgir argumentos suficientes para refinar, alterar, ou mesmo negar a teoria em questão. Em síntese, na medida por teoria deve haver uma circularidade evolutiva entre teoria-medida-teoria por duas razões fundamentais: a primeira é que, sem a estreita e contínua relação teoria-medi$\mathrm{da}$, as medidas aplicadas podem estar mensurando um construto que nada tem a ver com o pretendido, ou então, que podem estar medindo nada, nos casos em que o instrumento não parte de alguma articulação teórica minimamente consistente; a segunda razão é que, sem a estreita e contínua relação teoria-medida, não ocorre o refinamento da teoria e, por conseguinte, do conhecimento cientificamente $\operatorname{articulado}^{(5)}$.

Quando se trata de fenômenos aos quais não se aplicam medidas fundamentais e nem medidas derivadas, essa perspectiva teórica tem que ser mantida porque é a teoria que alicerça a medida. Ao se desenvolver um instrumento de medida, há menos interesse pelos itens do instrumento do que pelo construto que ele pretende medir ${ }^{(3)}$; os itens da escala são um meio para a avaliação do construto ${ }^{(6)}$.

A psicometria fundamenta-se teoria das medidas e, num sentido restrito, trata da medida de construtos ou traços latentes, representados por comportamentos observáveis ${ }^{(5)}$.

\section{SENTIMENTO DE IMPOTÊNCIA}

A North American Nursing Diagnosis Association (NANDA) reconheceu a resposta impotência - no original powerlessness - como pertinente à enfermagem e a incluiu em sua classificação em $1982^{(7)}$.

Na versão brasileira da Classificação da NANDA de $2002^{(8)}$ esse diagnóstico foi traduzido como impotência. Observações não sistemáticas nos têm indicado que freqüentemente esse termo é confundido com resposta pertinente à função sexual, o que nos motivou a sugerir que a tradução para a língua portuguesa seja modificada para sentimento de impotência. Entendemos que, enunciado dessa forma, o título do diagnóstico remete o conceito para a área psicossocial, distanciando-o da área de função sexual. Por essa razão, neste artigo, utilizaremos o termo sentimento de impotência para designar o original powerlessness.

\section{Sentimento de impotência foi definido como}

a percepção de que uma ação própria não afetará significativamente um resultado; uma falta de controle percebida sobre uma situação atual ou um acontecimento imediato(9).

A importância desse diagnóstico e sua presença nos pacientes são observadas na literatura em enfermagem. $\mathrm{O}$ sentimento de impotência é uma condição que pode afetar todas as pessoas ao longo de suas vidas e qualquer processo de doença, agudo ou crônico, pode predispor ao sentimento de impotência ${ }^{(10-11)}$.

As crianças hospitalizadas comumente experimentam o sentimento de impotência em relação ao ambiente hospita$\operatorname{lar}^{(12)}$. Nos idosos, a vulnerabilidade para esse sentimento aumenta devido às freqüentes perdas que geralmente experimentam e aos estressores psicológicos como a morte de um amado, aposentadoria, deslocamento e mudanças fisiológicas que acompanham o envelhecer.

O sentimento de impotência pode ocorrer também no indivíduo com doença aguda, especialmente durante as fases de acompanhamento diagnóstico, quando os sintomas são mais prevalentes, quando a doença é mais debilitante, quando o indivíduo não teve experiências com doenças ou quando a avaliação diagnóstica é prolongada ou muito invasiva $^{(13-15)}$.

Com a doença crônica, que impõe um longo período de adaptação, há aumento do risco de sentimento de impotên$\mathrm{cia}^{(16-17)}$. O paciente pode experimentar flutuações no estado de saúde, com o início de deterioração, com os procedimentos diagnósticos e tratamentos invasivos, eventos que podem alterar a ele próprio e a seu estilo de vida.

Outros fatores também contribuem para o sentimento de impotência do paciente: o meio atual de cuidados de saúde, a tecnologia e a linguagem pouco ou nada familiares, sua privacidade e suas necessidades muitas vezes ignoradas, assim como a remoção de seus objetos pessoais. $\mathrm{O}$ acesso ao paciente é limitado, a vigilância constante e o uso de equipamentos em seu território são freqüentes. Também as interações pessoais podem desencorajar o envolvimento do paciente no tratamento, reforçando fracassos pessoais prévios, o que predispõe ao senso de fragilidade e diminui o senso de controle sobre $\mathrm{si}^{(13,18)}$.

O sentimento de impotência percebido pelo paciente guarda estreita relação com o ambiente de cuidados que pode favorecer ou, de certa forma, impor essa percepção, e os cuidados de enfermagem são elementos importantes para que os pacientes desenvolvam maior ou menor percepção de controle das situações ${ }^{(14)}$. A supervisão contínua que as enfermeiras realizam, levando o paciente a não assumir responsabilidades em seus próprios cuidados, pode provocar diminuição da auto-estima e sentimento de impotência ${ }^{(19)}$.

Essas considerações indicam que o sentimento de impotência é um construto pertinente à clínica de enfermagem, o que justifica investimentos no sentido de desenvolve-lo e refiná-lo. Como um diagnóstico, o sentimento de impotência tem alto grau de abstração e, além disso, compartilha seus indicadores com outros diagnósticos, o que torna difícil a tarefa de identificá-lo de forma acurada na clínica. De- 
senvolver e refinar esse diagnóstico trará elementos para que ele seja aplicado de forma adequada.

O sentimento de impotência é uma resposta psicossocial, subjetiva, complexa, envolve fatores multidimensionais que se inter-relacionam como, por exemplo, fatores culturais, características da personalidade, experiências pessoais, fatores cognitivos e emocionais. Com isso, pode-se dizer que o sentimento de impotência é um construto que não pode ser mensurado diretamente. No entanto, como construto, apresenta atributos que podem ser mensurados por teoria, e a psicometria é um referencial teórico-metodológico adequado para esse fim.

\section{REFERENCIAL TEÓRICO- METODOLÓGICO PARA O DESENVOLVIMENTO DE MEDIDAS}

O ponto de partida para a construção de instrumentos para medir uma variável psicosssocial é a operacionalização de seus indicadores clínicos. Como outras variáveis psicossociais o sentimento de impotência é uma resposta complexa e com alto grau de abstração. Para mensurá-la, faz-se necessário, portanto, movê-la do abstrato para o concreto, através da identificação e operacionalização das propriedades, ou atributos, que caracterizam esse construto. Nesse contexto, operacionalizar atributos significa especificar indicadores empíricos que serão usados para comunicar um significado, assim como os procedimentos que serão usados para medi-lo. O foco da mensuração é quantificar as características do conceito a ser mensurado e a psicometria, fundamentada nas teorias da medida, guia o alcance desse foco.

O sentimento de impotência, como uma variável de interesse para a enfermagem clínica, é um conceito que não pode ser observado diretamente, mas é representado por comportamentos observáveis. Considerando a estreita relação teoria-medida, a aplicação da psicometria ao conceito de sentimento de impotência pode fornecer argumentos que fundamentarão o refinamento desse construto, bem como maior acurácia em sua aplicação na prática clínica.

A construção de um instrumento, segundo a psicometria, tem como base a explanação teórica do construto que se quer medir. Essa explanação teórica deve permitir enunciar as propriedades que caracterizam o construto em questão. Com base nessas propriedades são definidos comportamentos observáveis que as representam que, por sua vez, darão origem aos possíveis itens do instrumento de medida. Um item pode ser uma frase, uma afirmação cujo conteúdo expressa um atributo do construto. $\mathrm{O}$ conjunto de itens do instrumento deve representar o conjunto de atributos do construto em estudo. A demonstração do grau com que essa representação ocorre fundamenta-se na análise de resultados da aplicação empírica do instrumento. Essa demonstração deve ser feita por análises estatísticas dos itens individualmente e do conjunto de itens como um todo ${ }^{(5)}$. Para tanto, foi desenvolvida uma série de parâmetros mínimos que a medida psicométrica deve apresentar para ser legítima. Esses parâmetros referem-se à análise dos itens (dificuldade e discriminação), à validade e à confiabilidade (fidedignidade) do instrumento.

Um instrumento é válido quando, de fato, mede o que supostamente deveria medir; ou seja, ao se medirem os itens, que são a representação comportamental do construto, mede-se o próprio construto. Para a demonstração da validade de um instrumento, utilizam-se técnicas diversas e, a rigor, a validação de uma medida é um processo cumulativo.

A confiabilidade da medida é apresentada sob uma série heterogênea de nomes: precisão, fidedignidade, estabilidade, constância, equivalência e consistência interna $^{(5)}$. A fidedignidade ou precisão de um teste diz respeito à característica que ele deve possuir - a de medir sem erros - razão dos nomes precisão, confiabilidade e fidedigni$\operatorname{dade}^{(5)}$.

Esses são alguns dos principais fundamentos teóricos do método de construção de instrumento para medir variáveis psicos-sociais. O método é composto por três conjuntos de procedimentos: teóricos, empíricos ou experimentais e analíticos (estatísticos) $)^{(4)}$.

A seguir, apresentaremos uma breve explicação de cada procedimento ${ }^{(4-5)}$.

No pólo teórico, é enfocada a explicitação da teoria sobre o construto para o qual se quer desenvolver um instrumento de medida e a operacionalização do construto em itens. Nesse pólo, o pesquisador que desenvolve o instrumento deve explicitar a teoria sobre a variável em questão, sobre o construto. Como parte dessa explicitação, deve também identificar os comportamentos que constituem uma representação adequada do mesmo construto. Geralmente, esses comportamentos são expressões verbais capazes de caracterizar a presença ou a magnitude da variável em estudo. Na explicitação teórica do construto, o pesquisador deve considerar também a dimensionalidade do construto que pode ser uni ou multidimensional. Essa definição deve ser explorada no pólo teórico de modo que o construto fique o mais claro possível e que seja suficientemente preciso para a construção dos itens do instrumento de medida. Nesse pólo estão implícitas as definições constitutivas e operacionais do conceito. Definir constitutivamente um construto significa definir os termos, a semântica do 
construto. Definir operacionalmente um construto diz respeito à elaboração dos itens, expressões da representação comportamental do construto.

Ao se elaborarem os itens, há alguns critérios a serem observados para que o construto seja bem representado. A elaboração dos itens é seguida por análises teóricas que têm a finalidade de julga-los quanto a aspectos semânticos, aspectos de inteligibilidade e quanto à pertinência dos itens ao construto ${ }^{(4)}$.

No caso do desenvolvimento de um instrumento para a mensuração do sentimento de impotência o pólo teórico constituiu-se da análise de publicações de pesquisas e textos didáticos que tratavam do assunto. Nesse processo observou-se que outros construtos fazem interface com o de sentimento de impotência e, por vezes, a ele se surperpõem, como por exemplo, a desesperança, auto-eficácia, ansiedade e auto-estima. Realizou-se então a revisão desses conceitos frente às definições encontradas de sentimento de impotência com o propósito de identificar os limites do construto de sentimento de impotência.

Na literatura sistematicamente pesquisada foi identificado um modelo representativo do sentimento de impotência $^{(15)}$. Apesar de esse modelo não ter sido desenvolvido após a sua proposição, julgou-se que ele era suficientemente consistente com o restante da literatura sobre o assunto, que permitia razoável delimitação do sentimento de impotência frente aos outros construtos com que faz interface, que era pertinente à perspectiva de saúde-doença e consistente com a definição de sentimento de impotência da North American Nursing Diagnosis Association (NANDA). Como resultado dos procedimentos do pólo teórico, assumiu-se o modelo de White, Roberts ${ }^{(15)}$ para nortear a construção do instrumento. Nesse ponto de desenvolvimento havia clareza de que o instrumento a ser construído serviria também para confirmar, ajustar ou negar esse modelo teórico.

Neste modelo ${ }^{(15)}$ propõem-se quatro dimensões de perda de controle pessoal como componentes do conceito de impotência: fisiológica, cognitiva, ambiental e decisória. A Figura 1 representa essa proposta.

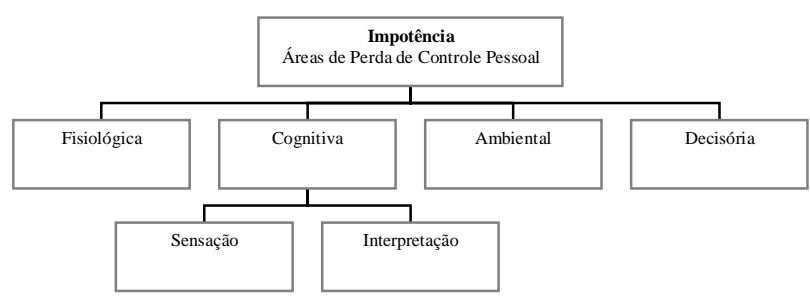

Figura 1 - Model o de I mpotência

Fonte: White BS, Roberts SL. Powerlessness and the pulmonary alveolar edema patient. Dimens Crit Care Nurs. 1993;12(3):129.
Descrevem-se, a seguir, as dimensões da impotência, conforme a proposta ${ }^{(15)}$. A perda de controle fisiológico refere-se aos sintomas físicos que, muitas vezes, impõem limites que a pessoa não pode controlar. A perda de controle cognitivo está presente quando o paciente não consegue compreender as sensações físicas que experimenta. Envolve a avaliação que o indivíduo faz e as conclusões a que chega a partir das informações obtidas pelos sentidos. $\mathrm{O}$ controle cognitivo refere-se tanto à sensação quanto à avaliação. $\mathrm{O}$ controle cognitivo sensorial consiste na percepção de ter poder para criar uma imagem mental e sobre que sensações esperar dos eventos que estão ocorrendo. A total falta de conhecimento de que sensações esperar origina sentimentos de impotência. O controle cognitivo referente à interpretação capacita o paciente a focalizar a atenção no evento ameaçador, buscar pistas sobre um possível dano e enfatizar a qualidade da experiência. Quando o paciente obtém informações sensoriais, ele as interpreta e faz uma avaliação subjetiva de seu controle sobre a situação.

A perda de controle ambiental é a percepção que a pessoa tem de não poder controlar um espaço físico que considera como seu território. $\mathrm{O}$ ambiente lhe é estranho e, se eventualmente conhecido, pode parecer-lhe hostil e impessoal. Percebendo-se incapaz de explorar o meio ou mudá-lo por imposição de outros, ou por qualquer outra razão, o paciente pode perceber-se impotente.

A perda de controle decisório ocorre quando a pessoa não tem mais o poder para tomar decisões sobre si e seu cuidado. O controle decisório depende da diversidade de opções de escolha em determinada situação e do "poder escolher".

Nas dimensões desse modelo de impotência está sempre presente a idéia de perda de controle. Esse modelo teórico foi assumido como a base para construir um instrumento de medida do sentimento de impotência. A definição constitutiva de sentimento de impotência para o instrumento é: um estado em que a pessoa percebe perda de controle pessoal sobre como lidar com as limitações físicas decorrentes de problemas de saúde, sobre o ambiente físico de cuidados à saúde, sobre as decisões relativas à própria saúde ou sobre os significados das sensações físicas que experimenta. Essa definição constitutiva circunscreve o sentimento de impotência ao contexto de saúde-doença.

A análise do construto, que deve sustentar uma definição constitutiva como já apresentado, é a base para a definição operacional que se refere à construção dos itens capazes de expressar a representação comportamental do construto. Considerando a definição constitutiva e cada dimensão do sentimento de impotência, tal como definidas no modelo adotado, o passo seguinte é a criação de enunciados representativos desse sentimento. Construíram-se, então, frases cujos conteúdos seriam pertinentes à percepção de controle pessoal sobre a saúde nas dimensões 
cognitiva, ambiental, fisiológica e decisória. Essa construção, inicialmente, é realizada sem limite em termos de quantidade de itens, num esforço de se obter a representação de toda a amplitude de cada dimensão. Já nessa fase deve-se levar em conta o perfil da população a que o instrumento se destinará e o tipo de escala de respostas que se desejará e se o instrumento será auto-aplicável ou aplicável por entrevista. Sabe-se que nas etapas seguintes os itens serão crivados, selecionando-se os que melhor atendem critérios de pertinência às dimensões do construto, de adequação semântica e de inteligibilidade. O julgamento dos itens é realizado pelo exame cuidadoso de cada um deles por um painel de especialistas que, além de experiência com o conceito, devem também ter familiaridade com a psicometria.

A partir do julgamento dos especialistas, os melhores itens, segundo os critérios estabelecidos, são selecionados e os outros são descartados. Os itens selecionados são então apresentados a sujeitos, ou grupos de sujeitos, com características semelhantes aos da população alvo. Apresentam-se a eles os itens, um a um, e pede-se que eles os reproduzam com as suas próprias palavras, permitindo que teçam comentários sobre os conteúdos dos itens. O pesquisador observa e registra se algum item é mal compreendido, se há dificuldade em entender o que querem dizer, se os sujeitos precisam, sistematicamente, de esclarecimentos adicionais. Com as observações que vai obtendo, o pesquisador vai ajustando os itens e reapresentando-os a outros sujeitos, até que não haja mais indicativos de necessidade de outros ajustes. Durante essas discussões, pode-se também avaliar a adequação das orientações para responder o instrumento, caso ele seja auto-aplicável, assim como a compreensão sobre como trabalhar com as alternativas de respostas (escala de frequiencia, de intensidade, de concordância, numérica, visual análoga etc). Os itens que resistirem até o final dessa etapa são então preparados para compor um instrumento piloto. Essa preparação inclui decidir a ordem dos itens e a formatação propriamente dita, considerando detalhes que podem facilitar a obtenção de respostas válidas e completas. Nos pólos subseqüentes - empírico e analítico - esse instrumento piloto é submetido a testes e análises que fornecem outros indicadores de propriedades de cada item e estimativas de validade e confiabilidade do instrumento como um todo.

No pólo empírico ou experimental definem-se as etapas e técnicas da aplicação do instrumento piloto e da coleta da informação para proceder à avaliação da qualidade psicométrica do instrumento ${ }^{(4)}$. Nesse pólo seleciona-se a população e define-se a amostra dos sujeitos que serão convidados a participar do estudo. É importante que a amostra escolhida permita ampla variação de respostas. O tamanho da amostra deve ser estimado de acordo com as exigências das análises estatísticas planejadas. Como regra geral, para a análise fatorial, recomenda-se o cálculo com base em 10 sujeitos por item ${ }^{(20)}$. Assim, se o instrumento piloto ficou com, por exemplo, 20 itens o ideal é um total de 200 respostas. Os procedimentos de coleta dos dados devem favorecer a obtenção de respostas completas e válidas. É melhor despender mais tempo para a coleta dos dados que obter respostas incompletas ou com validade questionável. São coletados também dados para caracterização da amostra, escolhendo-se variáveis que permitam futuras comparações de resultados. Os dados coletados são verificados quanto à completude e lançados em planilhas ou banco de dados eletrônicos de acordo com plano de análises e testes estatísticos que serão realizados no pólo analítico.

O pólo analítico inclui os procedimentos de análises estatísticas a serem efetuadas sobre os dados para estimar a validade e confiabilidade do instrumento produzido e, se pertinente, para estabelecer sua normatização. Esses três conjuntos de procedimentos indicarão a solidez psicométrica da ferramenta e permitirão avaliar a coerência do seu conteúdo com o conceito - sentimento de impotência ${ }^{(4)}$. Há diversas formas para se estimar a validade e confiabilidade de instrumentos de medidas e apresentá-las foge ao objetivo deste artigo. O pesquisador que pretende trabalhar com o desenvolvimento de instrumentos de medida deve se reportar à vasta literatura sobre o assunto, antes de iniciar o empreendimento. É possível que as análises estatísticas indiquem a inadequação ou a irrelevância de algum item do instrumento piloto. Nesse caso, o pesquisador é que vai decidir pela manutenção ou exclusão de itens. Se houver a decisão por exclusão de itens, as análises de confiabilidade e validade são repetidas com os itens que finalmente permanecerem no instrumento.

Se os índices de confiabilidade e as estimativas de validade forem aceitáveis, o pesquisador deve retornar ao modelo teórico para verificar a consistência entre ele e os resultados que obteve com o instrumento. Isso pode mostrar limitações atribuíveis à teoria ou à medida. O próprio pesquisador deve identificar as possíveis limitações e apresenta-las à comunidade profissional que disporá de um instrumento em fase inicial de desenvolvimento. Isso significa que o processo de validação do instrumento é contínuo; hipóteses sobre o construto medido pelo instrumento devem ser submetidas a testes em outros estudos, construindo-se a circularidade evolutiva entre a medida e a teoria que a fundamenta.

\section{CONSIDERAÇÕES FINAIS}

Os procedimentos da psicometria são extremamente úteis para o desenvolvimento do conhecimento da enfermagem. 
A disponibilidade de instrumentos para avaliar respostas psicossociais, tão freqüentes no cenário clínico, contribuirá para a acurácia dos diagnósticos que as enfermeiras realizam, para a validação clínica desses diagnósticos e para o teste de intervenções.

A experiência de iniciar a construção de um instrumento para avaliar o sentimento de impotência mostrou que os procedimentos mais árduos são os do pólo teórico. Exigem

\section{REFERÊNCIAS}

(1) Pedhazur EJ, Schmelkin LP. Measurement, design, and analysis: an integrated approach. Hillsdale (NJ): Lawrence Erlbaum; 1991

(2) Rempusheski VF. The proliferation of unreliable and invalid questionnaires. Appl Nurs Res. 1990;3(4):174-6.

(3) Pett MA, Lackey NR, Sullivan JJ. Making sense of factor analysis. Thousand Oaks: Sage; 2003.

(4) Pasquali L. Princípios de elaboração de escalas psicológicas. Rev Psiquiatr Clín. [periódico on line] 1998;25(5 ed. esp): 206-23. Disponível em: URL: http://www.hcnet.usp.br/ipq/ revista/r255/conc255.htm> (Acesso em 04 jul. 2003).

(5) Pasquali L. Psicometria: teoria e aplicações. Brasília: Editora UnB; 1997.

(6) DeVellis RF. Scale development: theory and application. Newbury Park (CA): Sage; 1991

(7) Gordon M. Nursing diagnosis: process and application. $3^{\text {rd }}$ ed. Saint Louis: Mosby; 1994.

(8) North American Nursing Diagnosis Association (NANDA). Diagnósticos de enfermagem da NANDA: definições e classificação 2001-2002. Porto Alegre: Artmed; 2002.

(9) North American Nursing Diagnosis Association (NANDA) International. Nursing Diagnoses: definitions and classification 2003-2004. Philadelphia; 2003.

(10) Lambert VA, Lambert CE. Role theory and the concept of powerlessness. J Psychosoc Nurs Ment Health Serv. 1981;19(9):11-4. desenvolver a sensibilidade teórica, exercitando-a num constante ir e vir entre o mais concreto e o mais abstrato; exige desenvolver a tolerância à ambigüidade, sem abandonar o exame das evidências. Espera-se que o conteúdo deste artigo motive os pesquisadores a aprofundar conhecimentos sobre a psicometria para aplicá-los não só no desenvolvimento de medidas, mas também na escolha de instrumentos para medir variáveis de pesquisa que não podem ser medidas diretamente.
(11) Kubsch S, Wichowski HC. Restoring power through nursing intervention. Nurs Diagn. 1997;8(1):7-15.

(12) Carpenito LJ. Diagnósticos de enfermagem: aplicação à prática clínica. $8^{\text {a }}$ ed. Porto Alegre: Artmed; 2002.

(13) McFarland GK, McFarlane EA. Nursing diagnosis and interventions: planning for patients care. Saint Louis: Mosby; 1989.

(14) Boeing MH, Mongera CO. Powerlessness in critical care patients. Dimens Crit Care Nurs. 1989;8(5):274-9.

(15) White BS, Roberts SL. Powerlessness and the pulmonary alveolar edema patient. Dimens Crit Care Nurs. 1993;12(3):127-37.

(16) Benjamin D. Powerlessness in chronic illness. Prairie Rose 1996;65(4):9a-10a.

(17) Miller JF. Coping with chronic illness: overcoming powerlessness. $2^{\text {nd }}$ ed. Philadelphia: Davis; 1992.

(18) Efraimsson E, Rasmussen BH, Gilje F, Sandman P. Expression of power and powerlessness in discharge planning: a case study of an older women on her way home. J Clin Nurs. 2003;12(5):707-16

(19) Arakelian M. An assessment and nursing application of the concept of locus of control. ANS Adv Nurs Sci. 1980;3(1): $25-42$.

(20) Nunnaly JC, Bernstein IH. Psychometric theory. $3^{\text {rd }}$ ed. New York: McGraw-Hill; 1994. 